\title{
An Unusually Large Foreign Body in Oesophagus in a 2-Year-Old Male Child
}

\author{
Ajinkya Kelkar ${ }^{*}$, Kalpesh Patil² \\ ${ }^{1}$ Department of Otolaryngology and Head and Neck Surgery, Yashwantrao Chavan Hospital, Pune, Maharashtra, \\ India \\ ${ }^{2}$ Department of Paediatric Surgery, Yashwantrao Chavan Hospital, Pune, Maharashtra, India \\ Email: ${ }^{*}$ drajinkyakelkar@gmail.com
}

Received 1 May 2015; accepted 24 May 2015; published 27 May 2015

Copyright (C) 2015 by authors and Scientific Research Publishing Inc.

This work is licensed under the Creative Commons Attribution International License (CC BY). http://creativecommons.org/licenses/by/4.0/

(c) (i) open Arcess

\section{Abstract}

A large variety of foreign bodies are swallowed by children, but the majority of those pass through the gastrointestinal tract without any adverse effects. The highest incidence of swallowed foreign bodies occurs in children between 6 months and 3 years. We reported to you an unusually large foreign body ingested by a 2-year-old male patient who underwent a rigid oesophagoscopy but the foreign body had to be removed by open surgical technique. It is advisable to have a multidisciplinary approach while dealing with such cases.

\section{Keywords}

Foreign Body, Screw, Oesophagoscopy, Gastrostomy

\section{Introduction}

Foreign bodies in the oesophagus are common in young children and the older age groups. The increased incidence of swallowed foreign bodies in children could be due to their natural propensity to gain knowledge by putting things in their mouth and inadequate control of deglutination as well as tendency to cry, cough or play during eating. Accidental ingestion is common in children due to their habit of putting things in the mouth. The most frequently swallowed foreign bodies in children include coins, safety pins, and toy parts. The peak age in children is between six months and three years.

Older age groups, particularly denture wearers who have less oral sensation, are more susceptible to foreign body ingestion. Fish bone, mutton bone and poorly chewed food are the common foreign bodies.

Since the muscular activity of the upper portion of the oesophagus is weak as compared with pharyngeal

"Corresponding author. 
musculature, foreign bodies are propelled in the hypopharynx and are more likely to lodge in the cricopharynx. The next most common site is just above the gastro-oesophageal junction.

Failure in identification and management of such foreign bodies results in complications like erosion, perforation, retropharyngeal abscess and pulmonary complications.

The objective of this case report is to have a multidisciplinary approach to foreign body removal so as to prevent the subsequent complications.

\section{Anatomy and Pathophysiology}

Oesophagus is a vertical muscular tube that extends from the hypopharynx to the stomach. It measures 23 to 25 $\mathrm{cm}$ in length in adult [1]. It begins at the lower border of cricoid cartilage at the level of sixth cervical vertebra. It terminates at the cardiac orifice of the stomach at the level of eleventh thoracic vertebra. Normally the lumen of the oesophagus is collapsed in a flattened or stellate pattern. The diameter is reduced at four points: the cricopharynx, crossing of aorta, crossing of left main bronchus and the diaphragm. The average distance from the upper incisor teeth is $16 \mathrm{~cm}, 23 \mathrm{~cm}, 27 \mathrm{~cm}$ and $38-40 \mathrm{~cm}$ respectively.

\section{Clinical Presentation and Diagnosis}

Up to $35 \%$ of paediatric patients with oesophageal foreign bodies are asymptomatic. Therefore the history surrounding the foreign body ingestion is extremely important. When and how it occurred, as well as description of the object and subsequent symptoms can give the surgeon, avaluable information. If available, a duplicate of the object can be helpful in choosing the most appropriate instruments for oesophagoscopy and foreign body removal.

Symptoms of foreign body vary with its position and make up. Upper oesophageal foreign bodies produce dysphagia and suprasternal pain on swallowing. With more distal foreign bodies, presentation is vague and orientation and level may not be describable. Rough objects may cause mucosal injury while passing into the stomach and can cause pain which subsides in about 24 hours. Persistent pain suggests that the foreign body has remained lodged. Large objects can obstruct the oesophagus causing salivation and regurgitation of any swallowed liquid including saliva. They may also compress the trachea due to their bulk causing dyspnea.

Disc batteries may lodge in the oesophagus and react with the mucosa resulting in oesophageal burn, perforation and mediastinitis. Such foreign bodies must be removed as soon as possible to reduce the possibility of caustic leak.

Any suspicion of foreign body, its presence must be proved or disproved. Radiologic studies are necessary. Radio-opaque foreign bodies can be visualized in most instances with postero-anterior and lateral neck films from the skull base to thoracic inlet. Non radiopaque objects may also be found, such as increase in the distance between the cervical vertebra and the trachea or air in the cervical oesophagus. Very small radiodense foreign bodies can be visualized on Computed Tomography (CT).

\section{Case Report}

A 2-year-old male child presented to our casualty with history of ingestion of a metallic screw 4 hours prior. Patient's relative gave history of inability to swallow any liquid or solid food since then. There was no evidence of any respiratory distress.

On examination, air entry was equal in bilateral lung fields. Patient underwent plain roentgenogram in anteroposterior view suggestive of radiodense long metallic foreign body extending from cervical to upper thoracic regionon the left side of trachea, most likely to be in the oesophagus. There was no evidence of pneumomediastinum and soft tissue emphysema (Figure 1 and Figure 2).

After taking a written informed consent for the procedure, patient underwent an emergency oesophagoscopy. Rigid oesophagoscope was passed and the foreign body could be visualized just below the level of cricopharynx. We attempted removal of the foreign body with forceps but could not grasp the head of the screw due to lack of space surrounding the foreign body. An attempt was made passing a dormia basket beyond the head of the screw but the screw was so large that it could not be removed endoscopically. The screw was then pushed distally towards the stomach. C-arm fluoroscopy was used intra operative to confirm the position of the foreign body and decision was taken to remove it by performing a gastrostomy (Figure 3). 


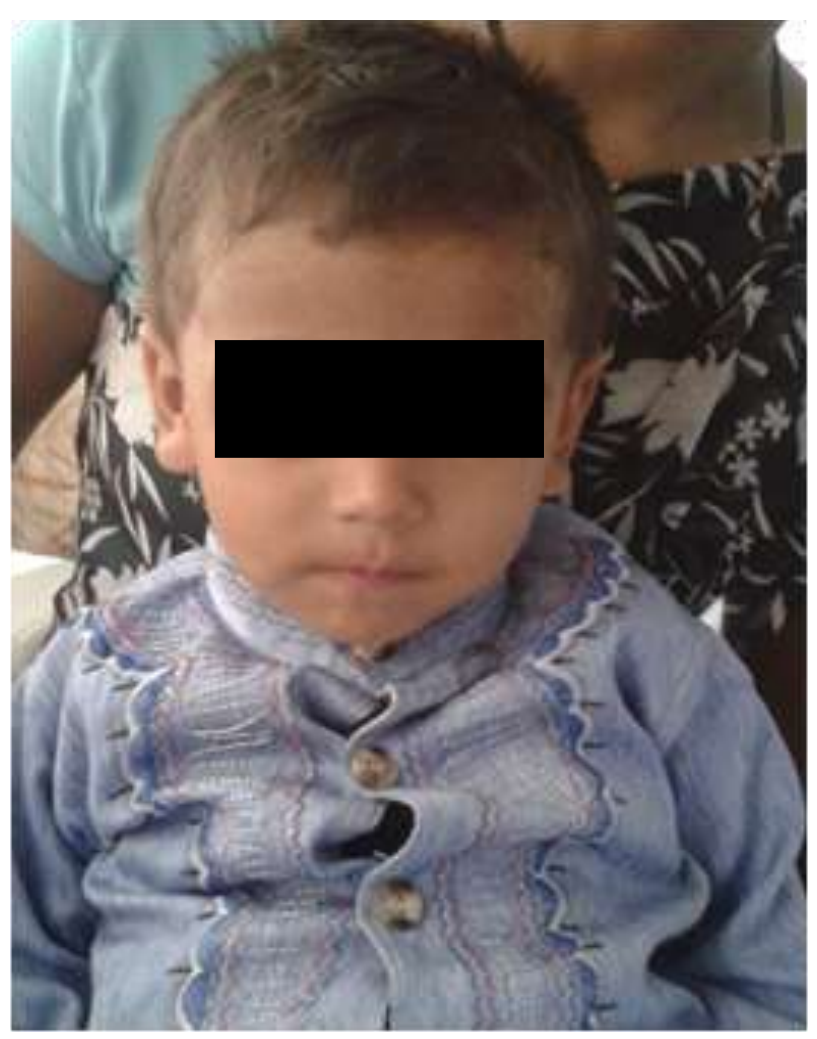

Figure 1. Clinical photograph of the patient.

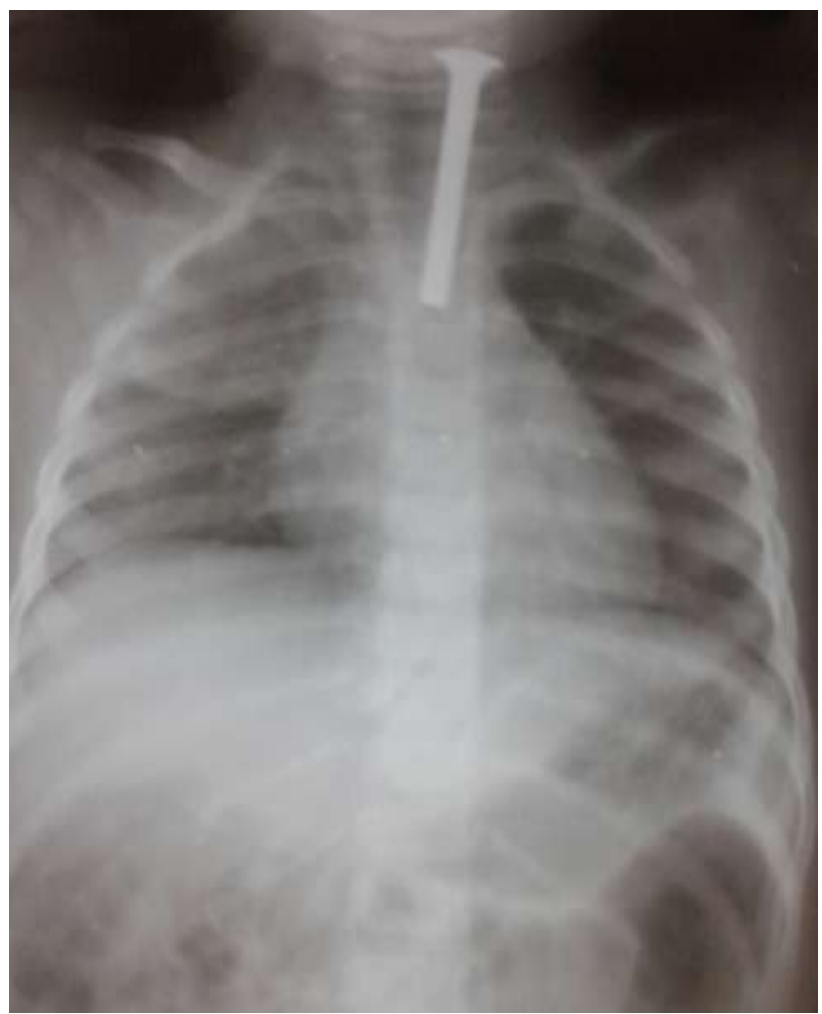

Figure 2. Plain roentgenogram in antero-posterior view in supine position. 
The case was handed over to our paediatric surgeon who performed a standard anterior gastrostomy through a left upper abdomen transverse incision. The screw could be palpated at the level of oesophago-gastric junction where the head of the screw remained engaged. By using Babcock's forceps $4.5 \mathrm{~cm}$ by $1 \mathrm{~cm}$ screw was finally retrieved. Gastrostomy was closed with 3 - 0 vicryl suture and abdominal incision closed in layers. Drain was inserted in the abdominal cavity. Patient was kept nil by mouth for 5 days and started on total parenteral nutrition. Drain was removed on fifth postoperative day after starting with clear oral feeds on the fourth post-operative day. Patient was discharged on seventh post-operative day (Figure 4 and Figure 5).

\section{Discussion}

A large variety of foreign bodies are swallowed by children, but the majority of those pass through the gastrointestinal tract without any adverse effect [2]. The highest incidence of swallowed foreign bodies occurs in children between 6 months and 3 years [3] [4]. Although 80\% to 90\% of swallowed foreign bodies will pass spontaneously, there is a definite predilection for swallowed foreign bodies to get impacted at the level of cricopharynx and just below it or at the oesophago-gastric junction.

Foreign bodies less than $2.5 \mathrm{~cm}$ in diameter and/or $<5 \mathrm{~cm}$ in length usually pass through whole gut. The level at which progress is impeded are pylorus, duodenum, duodenojejunoflexure. However any foreign body which is large or sharp may be impacted [5]. In our case, the foreign body was too large to negotiate through the constrictions of the gastro-intestinal tract and there were high chances of impaction and subsequent complications.

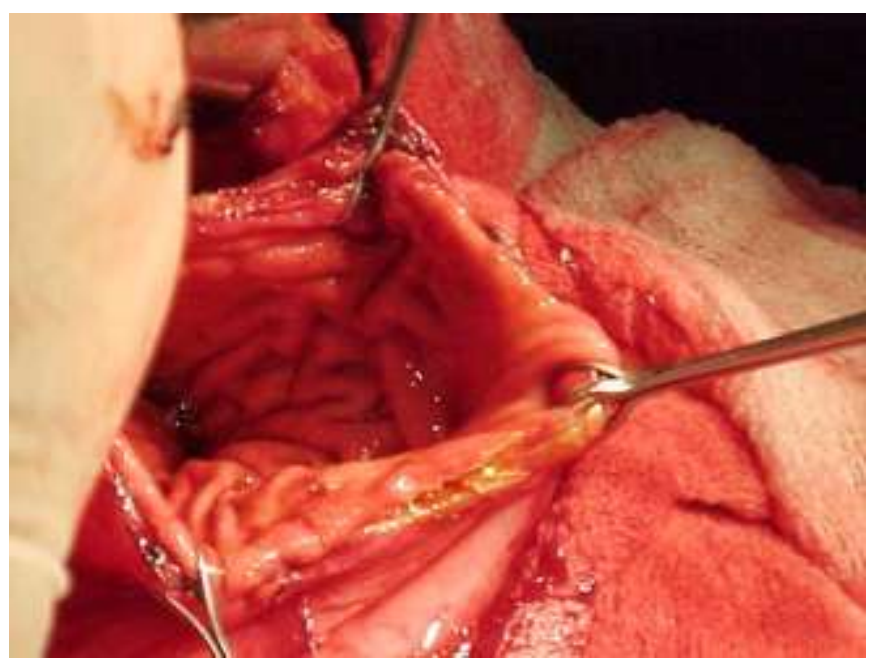

Figure 3. Anterior standard gastrostomy.

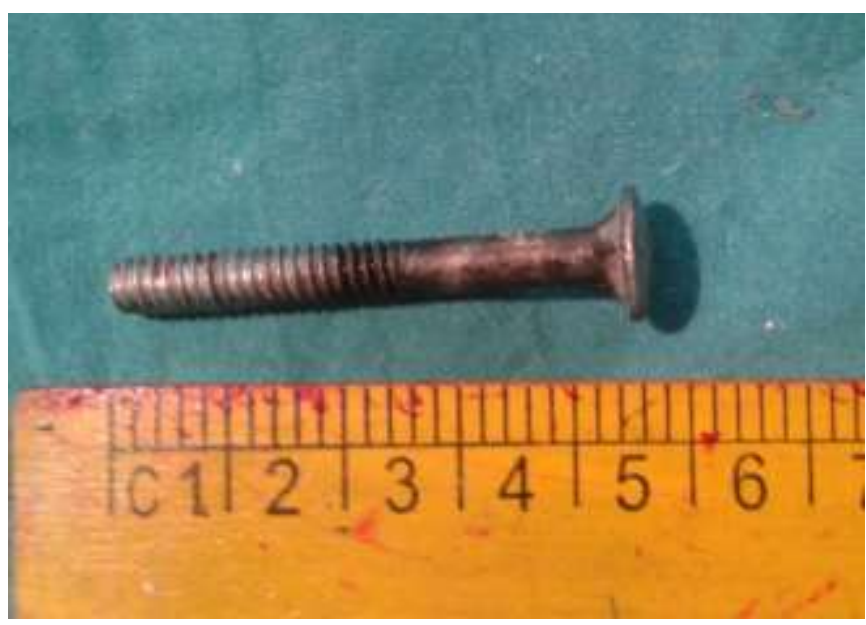

Figure 4. Foreign body (Screw) retrieved length $4.5 \mathrm{~cm}$. 


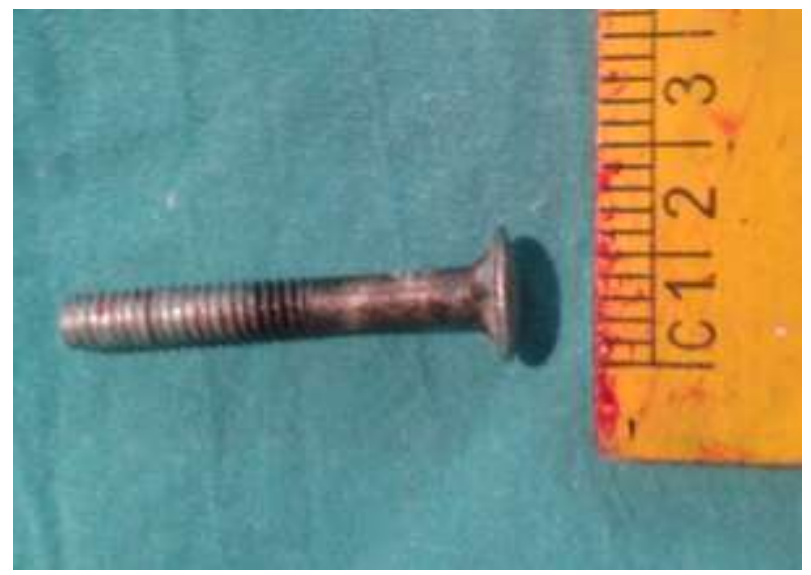

Figure 5. Foreign body (Screw) retrieved breadth $1 \mathrm{~cm}$.

Sharp foreign bodies like fish bone, chicken bone, sharp metallic wire, can be impacted anywhere from base of tongue to lower end of oesophagus. If they are not removed at the earliest, can cause complications such as a retropharyngeal abscess, ulcerative esophagitis, esophago-respiratory fistula, stricture formation, impaction and recurrent pneumonitis. Sharp foreign body can also be removed safely by endoscopic technique in most cases avoiding open surgical methods [6].

But in some cases, locating a sharp foreign body in pharynx or oesophagus is difficult. This may be due to light reflecting from the foreign body or major part of foreign body being hidden in the wall with only a small projection in the lumen. In such cases the C-arm fluoroscopy has to be utilized.

Sharp end of the foreign body has to be taken in the lumen of the endoscope to avoid complications. Partial dentures with sharp hooks, metallic springs, and screws are the most difficult and dangerous object to remove from oesophagus [7]. One can cause laceration and perforation during removal of such objects. Due to the high risk of complication, we took the decision of pushing the foreign body distally towards the stomach and retrieved it with open surgical technique.

\section{Conclusion}

Early diagnosis and immediate removal of foreign body are key to avoid any complications. Although $80 \%$ to $90 \%$ of the foreign bodies pass smoothly through the gastrointestinal tract, the nature of foreign body has to be determined. In case of a discbattery, it should be removed surgically if it remains in any one position for more than 24 hours. Sharp and large foreign bodies such as a screw have to be removed to prevent any further complications. It is advisable to have a team approach while dealing with such sharp and impacted foreign bodies.

\section{Disclosure}

None of the authors was given an honorarium, grant or other form of payment to produce this paper. We declare no potential conflict of interests, real or perceived.

\section{References}

[1] Schild, J.A. and Snow, Jr., J.B. (1996) Esophagology. In: Ballenger, J.J., Ed., Otorhinolaryngology Head and Neck Surgery, 15th Edition, Williams and Wilkins, USA, 1221-1235.

[2] Webb, W.A. (1988) Management of Foreign Bodies of the Upper Gastrointestinal Tract. Gastroenterology, 94, 204216.

[3] Nandi, P. and Ong, G.B. (1988) Foreign Body in the Esophagus: Review of 2394 Cases. British Journal of Surgery, 65 , 5-9. http://dx.doi.org/10.1002/bjs.1800650103

[4] Al-Salem, A.H., Qaisarrudin, S., Murugan, A., Hammad, H.A. and Talwalker, V. (1995) Swallowed Foreign Bodies in Children: Aspects of Management. Annals of Saudi Medicine, 15, 419-421.

[5] Tibbling, L. and Stenquist, M. (1991) Foreign Bodies in the Esophagus. A Study of Causative Factors. Dysphagia, 6, 224-227. http://dx.doi.org/10.1007/BF02493532 
[6] Sawant, P., Nanivadekar, S.A., Dave, U.R., Kanakia, R.R., Satarkar, R.P., Bhatia, R.S., et al. (1994) Endoscopic Removal of Impacted Foreign Bodies. The Indian Journal of Pediatrics, 61, 197-199. http://dx.doi.org/10.1007/BF02843619

[7] Holinger, L.D. (1990) Management of Sharp and Penetrating Foreign Bodies of the Upper Aerodigestive Tract. Annals of Otology, Rhinology \& Laryngology, 99, 684-688. http://dx.doi.org/10.1177/000348949009900902 